\title{
Wetting and prewetting of water on top of a single sheet of hexagonal boron nitride
}

\author{
M. C. Gordillo* \\ Departamento de Sistemas Físicos, Químicos y Naturales, Facultad de Ciencias Experimentales, Universidad Pablo de Olavide,

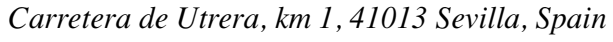 \\ J. Martí ${ }^{\dagger}$ \\ Departament de Física i Enginyeria Nuclear, Universitat Politècnica de Catalunya-Barcelona Tech, B5-209 Campus Nord UPC, \\ 08034 Barcelona, Catalonia, Spain
}

(Received 28 April 2011; published 6 July 2011)

\begin{abstract}
Wetting of a single hexagonal boron nitride sheet by liquid water has been investigated by molecular dynamics simulations within a temperature range between 278 and $373 \mathrm{~K}$. The wetting temperature was found to be $\sim 310 \mathrm{~K}$, while the onset of prewetting happens around the much higher temperature of $354 \mathrm{~K}$. The static (hydrogen-bond populations, density profiles, energy per molecule) and dynamic (diffusion coefficients) properties of water in the stable phases in this temperature range were also studied and compared to those of water on graphene. The results indicate that hydrophobicity of boron nitride is milder than that of graphene.
\end{abstract}

DOI: 10.1103/PhysRevE.84.011602

PACS number(s): 68.08.De, 61.30.Hn, 61.20.Qg, 31.15.xv

\section{INTRODUCTION}

Wetting can be regarded as the phenomenon that consists in the process of formation of a thin layer of adsorbent over a surface that at lower temperatures stays clean. Upon an increase in the density at the top of the surface, a first-order phase transition is produced from a thin toward a thick layer of the same substance [1-3]. The temperature at which the thin layer starts to form is called the wetting temperature $\left(T_{w}\right)$. At higher temperatures, we observe a different phenomenon: the difference in densities between the thin and thick layers decreases until it disappears at a temperature called the critical prewetting temperature $\left(T_{p w}\right)[4,5]$. For $T \geqslant T_{p w}$ there is a continuum layer formation upon an increase in density, instead of a first-order phase transition. An alternative way of viewing the wetting phenomena consists in monitoring the spreading of a single drop of liquid on the surface to form the thin layer described above through the variation of the contact angle of the water from a finite value to zero. The theoretical study of this phenomenon was pioneered by Young [6], while the relationship between criticality and wetting transitions was explored by Nakanishi and Fisher [7]. Reviews about wetting on different surfaces can be found in Refs. [8] and [9], among others.

Experimental evidence of wetting transitions started with simple gases such as hydrogen or helium located on alkali metal surfaces [10-13]. Later, studies of $\mathrm{Hg}$ on sapphire [14] and on molybdenum and niobium cells [15] reported wetting and prewetting transitions as well. The presence of defects and impurities at the surface is unavoidable, producing important difficulties to measure contact angles and modifying the behavior of liquids at interfaces. In the case of water, the contact angle with graphite has been measured to be between $60^{\circ}$ and $90^{\circ}$ at room temperature [16]. On the other hand, theoretical works have extensively analyzed the behavior of water close to surfaces, with a particular relevance of water-carbon interactions [17-19]. Among them some papers

\footnotetext{
*cgorbar@upo.es

†jordi.marti@upc.edu
}

evaluated the properties of water nearby boron nitride surfaces, such as nanotubes [20] or flat sheets [21]. The main aim of the present work is then to study the adsorption behavior of water on top of a single sheet of hexagonal boron nitride (h-BN), and from that, to derive its wetting and critical prewetting temperatures. In the rest of the paper, we will describe the model and methodologies employed in the simulations in Sec. II; the most important results about the wetting and prewetting transitions, together with additional information on dynamics of water, will be presented and discussed in Sec. III. Finally, we will briefly summarize the work in Sec. IV.

\section{METHOD}

We performed molecular dynamics calculations for a system that comprised a single $\mathrm{h}-\mathrm{BN}$ sheet and a variable number of water molecules on top of it (from 50 to 1000), at temperatures ranging between 278 and $373 \mathrm{~K}$. The B-N distance in this compound is similar but not identical to the $\mathrm{C}$-C distance in graphene (1.44 $\AA$ [22] instead of $1.42 \AA$ ), so our simulation cell was slightly bigger than the one we used in a previous work where water on graphene was simulated [23]: current values of $35.40 \times 35.04 \times 100 \AA$, instead of $34.4 \times$ $34.1 \times 100 \AA$ for the former. The length of the (normal to h-BN plane) $Z$ axis of the box was enough to ensure that the application of periodic boundary conditions was not necessary for this coordinate. The BN layer was located at $z=0$ and was kept frozen in all the calculations; i.e., the atoms on it were not allowed to move. A previous check on graphene [24] indicated that considering positional disorder did not change appreciably the adsorption properties of water. At each simulation, we kept constant the number of water molecules, the volume of the simulation cell, and the temperature.

As in previous works, water was modeled by a flexible simple point charge (SPC) potential [25], and the water-BN interactions were considered to be of the Lennard-Jones type [17]. The B- and N-oxygen and hydrogen parameters were obtained from Lorentz-Berthelot rules, taking as a starting point the B-B and N-N parameters from Ref. [26]. The results were $\epsilon_{\mathrm{BO}}=$ $61.11 \mathrm{~K}, \epsilon_{\mathrm{NO}}=75.49 \mathrm{~K}, \epsilon_{\mathrm{BH}}=20.27 \mathrm{~K}, \epsilon_{\mathrm{NH}}=25.03 \mathrm{~K}$, 


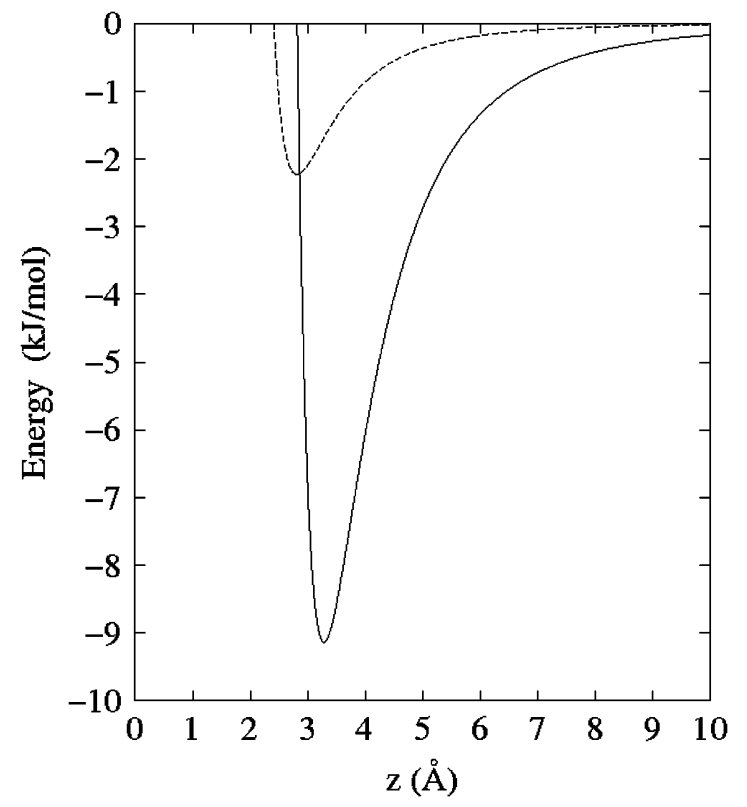

FIG. 1. Oxygen-h-BN (full curve) and hydrogen-h-BN (dashed curve) averaged-out potentials for a single h-BN layer.

$\sigma_{\mathrm{BO}}=3.31 \AA, \sigma_{\mathrm{NO}}=3.26 \AA, \sigma_{\mathrm{BH}}=2.84 \AA$, and $\sigma_{\mathrm{NH}}=$ $25.03 \AA$. These $\sigma$ values were similar to those for the $\mathrm{C}$-water interactions, but the $\epsilon$ ones are bigger by approximately $30 \%$ in the case of B-water interactions, and by $60 \%$ for the $\mathrm{N}$-water ones. We considered all the individual $\mathrm{B}$-water and $\mathrm{N}$-water interactions for each water molecule and all the atoms in the h-BN sheet. However, in order to visualize the strength of B-N layer-water interaction, we show an averaged-over version of these potentials in Fig. 1. The O-h-BN potential well considered in the present work is around $20 \%$ deeper than the one displayed in a previous simulation of a similar system [21]. This is probably due to the differences between the B-N distance used in that work $(1.53 \AA)$ and the experimental one [22] of $1.44 \AA$. This would account for most of the differences between the results of the present work and those reported in Ref. [21].

To complete our description, we employed a leap-frog Verlet integration algorithm coupled to a thermal bath in order to solve the equations of motion [27] and an integration time step of $0.5 \mathrm{fs}$. The equilibrations ran for at least $100 \mathrm{ps,} \mathrm{and} \mathrm{the}$ averages were calculated in runs of lengths between 125 and 250 ps. Further increasing the simulation time did not vary the averages of the properties we calculated.

To study the phenomena of wetting and prewetting we need to check the number of phases that are thermodynamically stable in a given temperature range, since the signature of wetting is the appearance of two stable phases of water on top of the considered surface. We did so by using the same technique already used in previous works for similar systems [23,24,28,29]; i.e., we proposed a reasonable expression for the water free energy as a function of density and temperature, $F(\rho, T)$ :

$F(\rho, T)=\sum_{i=0}^{3} \sum_{j=1}^{3} b_{i j} \rho^{i} T^{1-j}+$ ideal polyatomic gas terms and used the well-known relationship from thermodynamics

$$
E=-T^{2} \frac{\partial(F / T)}{\partial T}
$$

to obtain an expression for the energy depending on the same parameters than $F(\rho, T)$. Thus, we performed least-squares fits to the total energy (an observable obtained directly from our molecular dynamics simulations) to the expressions derived from Eq. (2), using coefficients and Eq. (1) to get $F(\rho, T)$.

\section{RESULTS}

We show in Fig. 2 the adsorption energy of water. The difference between the curves displayed is the temperature, which goes from $373 \mathrm{~K}$ (upper symbols) to $278 \mathrm{~K}$ (bottom ones). These were raw data used in the fits to obtain $F(\rho, T)$. We observe that in all cases there is a minimum for densities between 0.1 and $0.3 \AA^{-2}$. As can be seen in Fig. 3, this minimum is absent in the graphene-water curves. There the upper curve was taken from a simulation of water on graphene [23] and corresponds (as the h-BN one, full symbols), to a temperature of $298 \mathrm{~K}$. The dashed line is the result of the fit of the simulation results to the energy functional of Eq. (2) for temperatures between 278 and $298 \mathrm{~K}$. We observe that the increasing in the water layer $\epsilon$ parameters produces, in addition to the minimum, an increase in the absolute value of the adsorption energy. However, as the density increases, these curves tend to converge to a common value, which should be the one for bulk water. In fact, for the largest density shown, the energy difference is already less than $3 \%$.

The least-squares fit of the three lower curves of Fig. 2, corresponding to 278, 288, and $298 \mathrm{~K}$ in Eq. (2), produces the curve displayed in Fig. 4. Error bars (between 0.15 and $0.5 \mathrm{kcal} / \mathrm{mol}$ for all data) have been omitted for clarity purposes. There we can see the free energy in the range corresponding to densities between 0.125 and $0.7 \AA^{-2}$, even

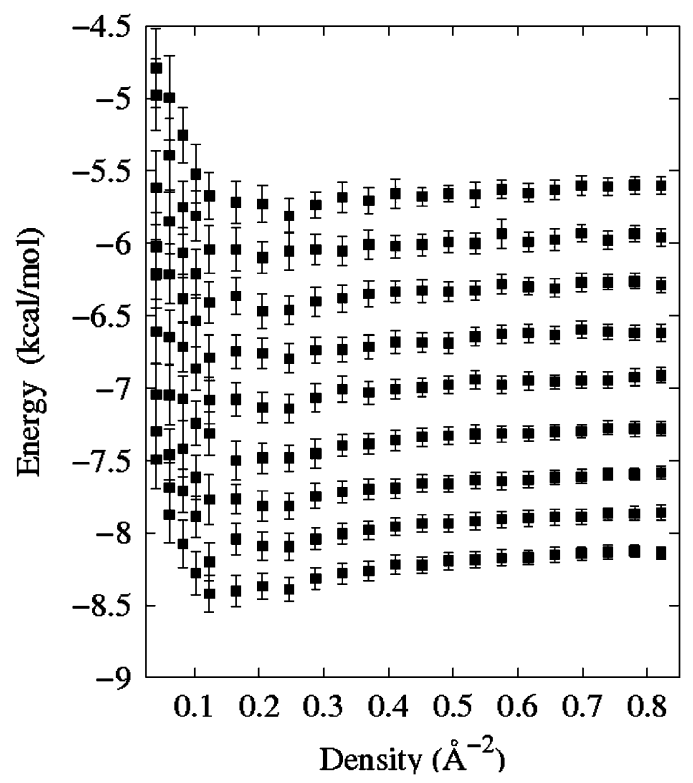

FIG. 2. Energy as a function of density for water on top of h-BN at several temperatures. From top to bottom, isotherms for 373, 360, $348,335,323,310,298,288$, and $278 \mathrm{~K}$. 


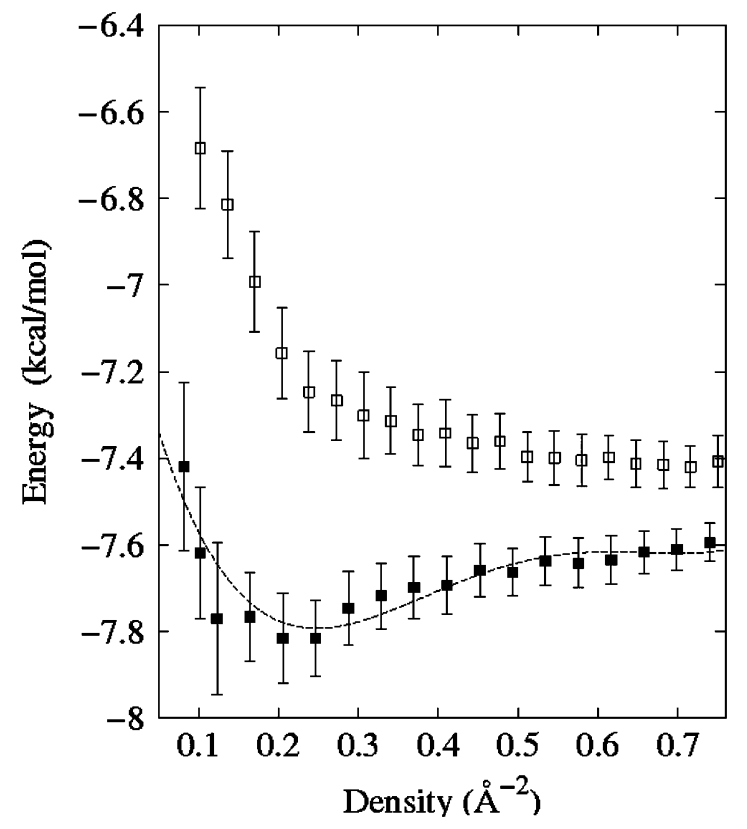

FIG. 3. Same than in Fig. 2, but for $T=298 \mathrm{~K}$ for water on graphene (open symbols) and on h-BN (full squares). The line is the result of the least-squares fit to the expression given in the text. The main difference is the existence of a minimum in the energy as a function of density in the second case.

though the fits were made starting at densities of 0.04 up to $0.82 \AA^{-2}$ (between 50 and 1000 water molecules in the simulation cell). The results indicate that the absolute minimum of the curve is at the lowest volume (inverse of the density) considered, indicating that in this temperature range, the bulk phase ( $\rho \rightarrow \infty$ in this scheme) will be always more stable than the

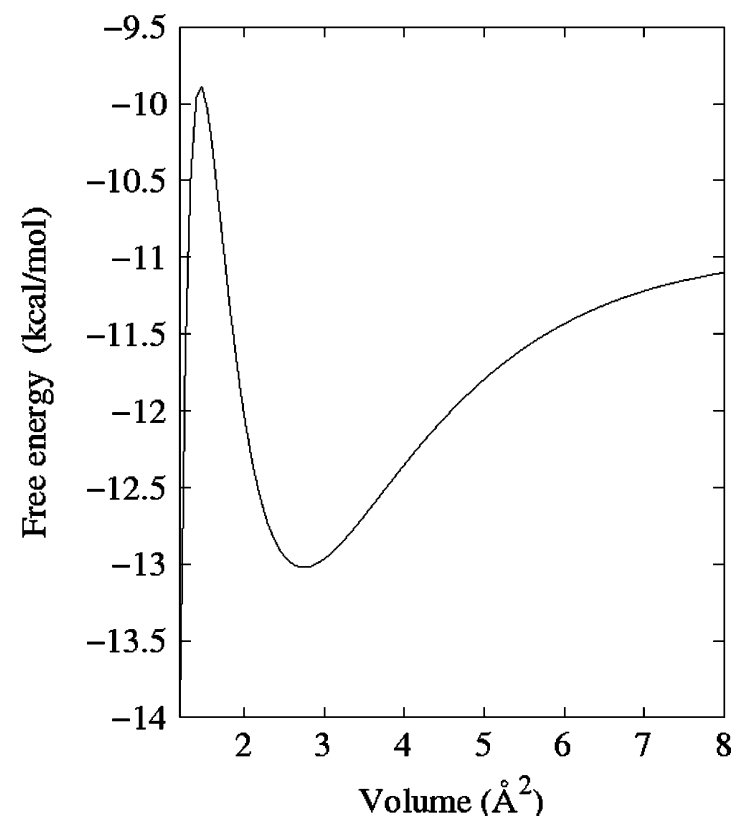

FIG. 4. Free energy as a function of water density for $T=298$ $\mathrm{K}$. The minimum of the curve is at volume $=0$, which corresponds to bulk water. See further explanation in the text. Estimations of error bars are in the range $[0.15,0.5] \mathrm{kcal} / \mathrm{mol}$.

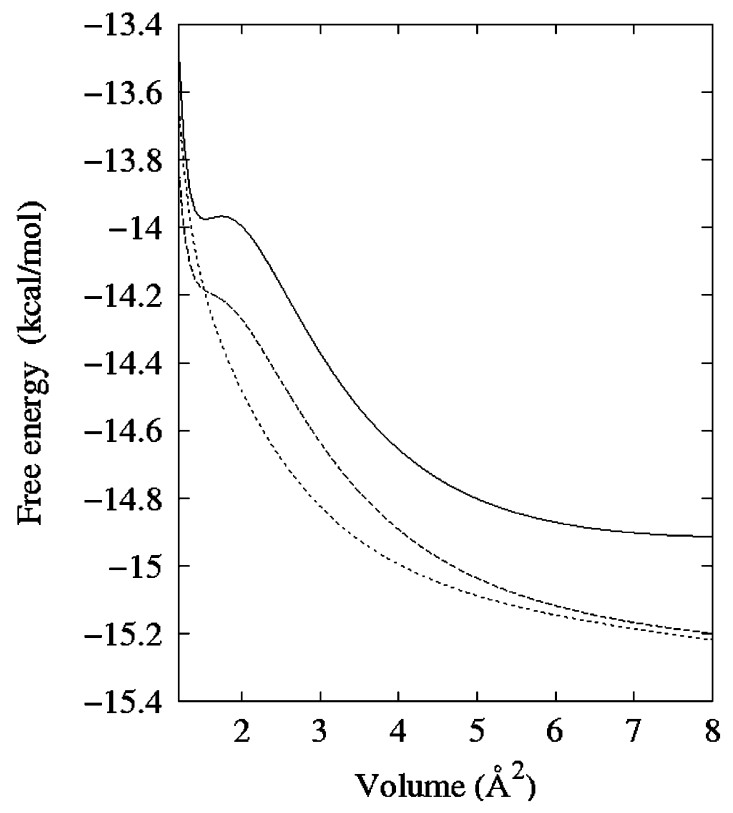

FIG. 5. Free energy as a function of water density for (from top to bottom), 316.5, 341.5, and 366.5 K. Error estimates as in Fig. 4.

adsorbed phase, represented by a local free-energy minimum at a volume equal to $2.74 \AA^{-2}$. All this means that water will not wet h-BN in this temperature range, and if we put some water on top of the h-BN layer, one or several drops will be formed.

Figure 5 gives the result of a similar fitting procedure for temperature ranges 310-323 K (full line), 335-348 K (dashed line), and 360-373 K (dotted line). Error estimates are in the same range as those in Fig. 4. These curves represent the free energies for the intermediate temperatures $316.5,341.5$, and $366.5 \mathrm{~K}$. What we observe is that the deep minimum at the lowest volume has disappeared, meaning that for $T>298 \mathrm{~K}$, water prefers to be on top of the h-BN surface rather than in bulk. In addition, the curves for 316.5 and $341.5 \mathrm{~K}$ are not monotonous functions of the density, as happens with the temperature of $366.5 \mathrm{~K}$. We can perform in both of them a Maxwell double-tangent construction to obtain two stable phases: a low-density one and a bulklike phase, for densities bigger than $0.7 \AA^{-2}$. The densities of the thinner phases are $0.29 \AA^{-2}$ for $316.5 \mathrm{~K}$ and $0.31 \AA^{-2}$ for $341.5 \mathrm{~K}$. This separation of phases is the signature of a wetting transition, with $T_{w} \sim 310 \mathrm{~K}$. This temperature is much lower than the one found in Ref. [21], where a value of $438 \pm 5 \mathrm{~K}$ was reported. As indicated before, this difference is due to the effective water-layer interaction, much deeper in the present work, due basically to the differences in the B-N distance, which decreases the adsorption energy of a single molecule from the $7.35 \mathrm{~kJ} / \mathrm{mol}$ of Ref. [21] to the $9.1 \mathrm{~kJ} / \mathrm{mol}$ of the present work. The effect of lowering the wetting temperature as the depth of the potential well increases was already reported in Ref. [19]. The rise in the adsorbate-surface interaction energy decreases the contact angle of the liquid on top of the solid (see, for instance, calculations for water on graphene in Ref. [19] for different graphene water potentials), which means that it is easier to reach the point at which the contact angle is $0^{\circ}$ (perfect wetting), decreasing the wetting temperature. The isotherm at 366.5 K (dotted line in Fig. 5) is a convex function of the 


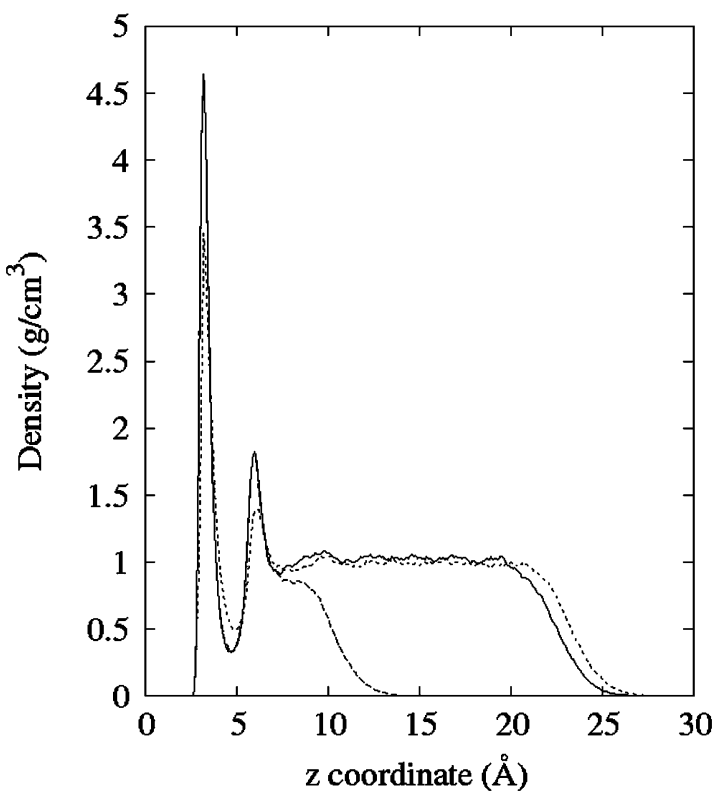

FIG. 6. Density profiles at $323 \mathrm{~K}$ for water on graphene at $0.72 \AA^{-2}$ (dotted line) and water on h-BN at $0.7 \AA^{-2}$ (full line) and $0.29 \AA^{-2}$ (dashed line).

inverse of the volume, indicating that in the temperature range from which it has been obtained (360-373 K), the width of the water layer increases regularly upon an increase in the density, with no phase transition at any point. This is the characteristic of a prewetting transition, and since the form of the curve is similar for $360 \mathrm{~K}$, we can say that the critical prewetting temperature is $T_{p w} \sim 354 \pm 6 \mathrm{~K}$ (average between the highest temperature of the previous isotherm and $360 \mathrm{~K}$ ). It should be noted that usually $T_{w}$ and $T_{p w}$ are connected by a continuous prewetting line [30].

Density profiles of water on top of h-BN at two densities $\left(0.29,0.7 \AA^{-2}\right)$ at $323 \mathrm{~K}$ are reported in Fig. 6 and compared with those of water on graphene at the highest density and the same temperature. Basically, what we see is that water behaves similarly when close to the surface in all cases. The only difference is that the heights of the peaks are biggest for water at h-BN, which indicates a less marked hydrophobic characteristics of this surface, when compared to a carbonbased one. The other noticeable difference concerns the length of the bulklike region of density $1 \mathrm{~g} / \mathrm{cm}^{3}$, which for water on graphene is somewhat longer than for water on h-BN, due precisely to the biggest peaks close to the surface.

The form of these density distributions is closely related to the percentages of hydrogen-bonded molecules (HB) as a function of the distance from the surface displayed in Fig. 7. Our definition of a $\mathrm{HB}$ is the same as in previous works $[17,31,32]$ and is based on geometrical criteria. So two water molecules are forming a HB when (1) the oxygen-oxygen distance is smaller than $3.6 \AA$, (2) the distance between the "acceptor" oxygen and the "donor" hydrogen is smaller than $2.4 \AA$, and (3) the $\mathrm{H}-\mathrm{O} \cdots \mathrm{O}$ angle is smaller than $30^{\circ}$. These conditions ensure a linear HB.

In the case of water close to h-BN at $323 \mathrm{~K}$ and density of $0.7 \AA^{-2}$ (Fig. 7) the amount of water molecules forming one or zero HBs is highest at the water-vacuum interface, as

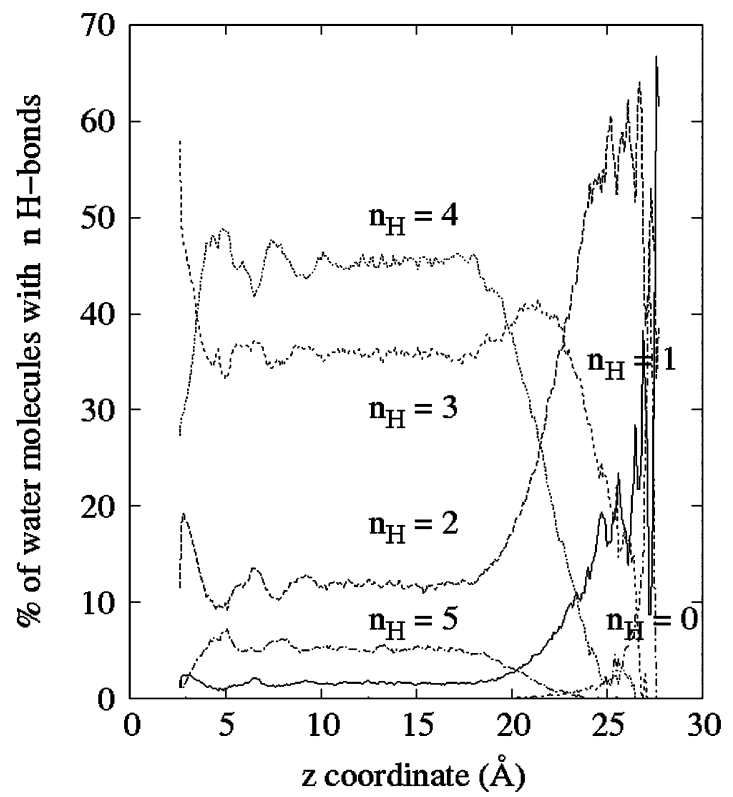

FIG. 7. Percentage of molecules with a $n_{H}$ hydrogen bonds as a function of the distance to the h-BN layer at $323 \mathrm{~K}$ and $0.7 \AA^{-2}$.

expected. Interestingly, the case $n_{H}=2$ shows a maximum for $z=25 \AA$, and its amount is bigger than $10 \%$ at any point of the system, whereas for $n_{H}=3$ we obtained important percentages over $35 \%$ in the bulklike region and even larger at the water-wall interface, as corresponds to water molecules with an $\mathrm{OH}$ pointing to the surface of the h-BN, something already reported for graphene [23]. Finally, the largest amounts of water forming four or five HBs are located at the central, bulklike region and are much lower at the water-BN interface. Our results are in good qualitative agreement with those of Dutta et al. [21] obtained with a similar model but different parameterization.

Some aspects of water dynamics have been also investigated. In particular, we obtained the self-diffusion coefficients $D$ through the slopes of the mean-square displacements of water molecules as a function of time; the results are reported in Table I.

We observe a quasimonotonic behavior for the two densities chosen: Generally, water diffusion rises with temperature (in a similar fashion as it happens in the bulk unconstrained system [33]) including some fluctuations, noticeably at the low density of $0.29 \AA^{-2}$. This result is essentially in good agreement with the findings of water adsorbed in graphite [34], indicating that water diffusion is not significantly influenced by the larger depth of the potential well in the present case.

TABLE I. Water self-diffusion coefficients $D$ (in $10^{-5} \mathrm{~cm}^{2} / \mathrm{s}$ ). Estimated errors are in parentheses.

\begin{tabular}{lcc}
\hline \hline Temperature $(\mathrm{K})$ & $0.29 \AA^{-2}$ & $0.7 \AA^{-2}$ \\
\hline 310 & $4.5(0.5)$ & $3.8(0.2)$ \\
323 & $5.5(0.6)$ & $4.7(0.2)$ \\
335 & $5.7(0.3)$ & $5.5(0.3)$ \\
348 & $7.6(0.8)$ & $6.2(0.4)$ \\
360 & $7.7(0.5)$ & $7.0(0.2)$ \\
\hline \hline
\end{tabular}




\section{CONCLUDING REMARKS}

The wetting behavior of water on top of a h-BN sheet has been investigated by molecular dynamics simulations. Waterwater interactions were modeled by a flexible SPC potential, whereas Lennard-Jones forces were responsible for modeling the forces between water and the BN surface. From the analysis of the phase diagram, we obtained that a wetting transition occurred at $T_{w} \sim 310 \mathrm{~K}$ and that critical prewetting would happen at $T_{p w} \sim 354 \pm 6 \mathrm{~K}$. The method employed to locate the wetting temperature is different from others described at the literature, based on the calculation of chemical potentials or on the determination of spreading coefficients [35], which require the knowledge of an interfacial probability distribution, not reported in the present work. However, we believe that the results obtained in the present work are meaningful and fully equivalent to those that could be obtained with the methods indicated above. Structural properties of such density profiles and the number of hydrogen bonds are qualitatively similar to those of water on graphene [23].

\section{ACKNOWLEDGMENTS}

MCG gratefully acknowledges financial support from the Spanish Ministry of Innovation and Science (grant FIS201018356) and the Junta de Andalucia (group PAI-205, and grant FQM-5985). JM thanks financial support from the Direcció General de Recerca de la Generalitat de Catalunya (grant 2009SGR1003) and the Spanish Ministry of Education and Science (grant FIS2009-13641-C02-01).
[1] J. W. Cahn, J. Chem. Phys. 66, 3667 (1977).

[2] C. Ebner and W. F. Saam, Phys. Rev. Lett. 38, 1486 (1977).

[3] X. Zhao, Phys. Rev. B 76, 041402(R) (2007).

[4] A. Hamraoui and M. Privat, J. Chem. Phys. 107, 6936 (1997).

[5] D. Bonn and D. Ross, Rep. Prog. Phys. 64, 1085 (2001).

[6] T. Young, Philos. Trans. R. Soc. London 95, 65 (1805).

[7] H. Nakanishi and M. E. Fisher, Phys. Rev. Lett. 49, 1565 (1982).

[8] P. G. de Gennes, Rev. Mod. Phys. 57, 827 (1985).

[9] D. Bonn, J. Eggers, J. Indekeu, J. Meunier, and E. Rolley, Rev. Mod. Phys. 81, 739 (2009).

[10] G. Mistura, H. C. Lee, and M. H. W. Chan, J. Low Temp. Phys. 96, 221 (1994).

[11] D. Ross, P. Taborek, and J. E. Rutledge, Phys. Rev. Lett. 74, 4483 (1995).

[12] D. Ross, J. A. Phillips, J. E. Rutledge, and P. Taborek, J. Low Temp. Phys. 106, 81 (1997).

[13] M. J. Bojan, G. Stan, S. Curtarolo, W. A. Steele, and M. W. Cole, Phys. Rev. E 59, 864 (1999).

[14] M. Yao and F. J. Hensel, J. Phys. Condens. Matter 8, 9547 (1996).

[15] V. F. Kozhevnikov, D. I. Arnold, S. P. Naurzakov, and M. E. Fisher, Phys. Rev. Lett. 78, 1735 (1997).

[16] M. E. Tadros, P. Hu, and A. W. Adamson, J. Colloid Interface Sci. 49, 184 (1974).

[17] M. C. Gordillo and J. Martí, Chem. Phys. Lett. 329, 341 (2000).

[18] G. Hummer, J. C. Rasaiah, and J. P. Noworyta, Nature (London) 414, 188 (2001).
[19] T. Werder, J. H. Walther, R. L. Jaffe, T. Halicioglu, and P. Koumoutsakos, J. Phys. Chem. B 107, 1345 (2003).

[20] C. Y. Won and N. R. Aluru, J. Phys. Chem. C 112, 1812 (2008).

[21] R. C. Dutta, S. Khan, and J. K. Singh, Fluid Phase Equilib. 302, 310 (2011).

[22] C. Jin, F. Lin, K. Suenaga, and S. Iijima, Phys. Rev. Lett. 102, 195505 (2009).

[23] M. C. Gordillo and J. Martí, Phys. Rev. B 78, 075432 (2008).

[24] M. C. Gordillo and J. Martí, J. Phys. Chem. B 114, 4583 (2010).

[25] J. Martí, J. A. Padró, and E. Guàrdia, J. Mol. Liq. 62, 17 (1994).

[26] D. Baowan and J. M. Hill, Micro. Nano Lett. 2, 46 (2007).

[27] H. J. C. Berendsen, J. P. M. Postma, W. F. van Gunsteren, A. Dinola, and J. R. J. Haak, J. Chem. Phys. 81, 3684 (1984).

[28] J. Martí, G. Nagy, M. C. Gordillo, and E. Guàrdia, J. Chem. Phys. 124, 094703 (2006).

[29] M. C. Gordillo and J. Martí, Phys. Rev. B 75, 085406 (2007).

[30] M. S. Sellers and J. R. Errington, J. Phys. Chem. C 112, 12905 (2008).

[31] J. Martí, J. Chem. Phys. 110, 6876 (1999).

[32] J. Martí, J. Sala, and E. Guàrdia, J. Mol. Liq. 153, 72 (2010).

[33] R. Hausser, G. Maier, and F. Z. Noack, Z. Naturforsch. A 21, 1410 (1966).

[34] G. Nagy, M. C. Gordillo, E. Guàrdia, and J. Martí, J. Phys. Chem. B 111, 12524 (2007).

[35] E. M. Grzelak and J. R. Errington, J. Chem. Phys. 132, 224702 (2010). 